\title{
Small antisense DNA-based gene silencing enables cell-free bacteriophage manipulation and genome replication - Supporting Information
}

Kilian Vogele†, Elisabeth Falgenhauer ${ }^{\dagger}$, Sophie von Schönberg ${ }^{\dagger}$, Friedrich C. Simmel ${ }^{\dagger}$, Tobias Pirzer ${ }^{*}$

† Physics of Synthetic Biological Systems-E14, Physics Department and ZNN, Technische Universität München, 85748 Garching, Germany

\author{
E-mail: pirzer@tum.de
}

Phone: +49 (0)89 289-11609. Fax: +49 (0)89 289-11612. 


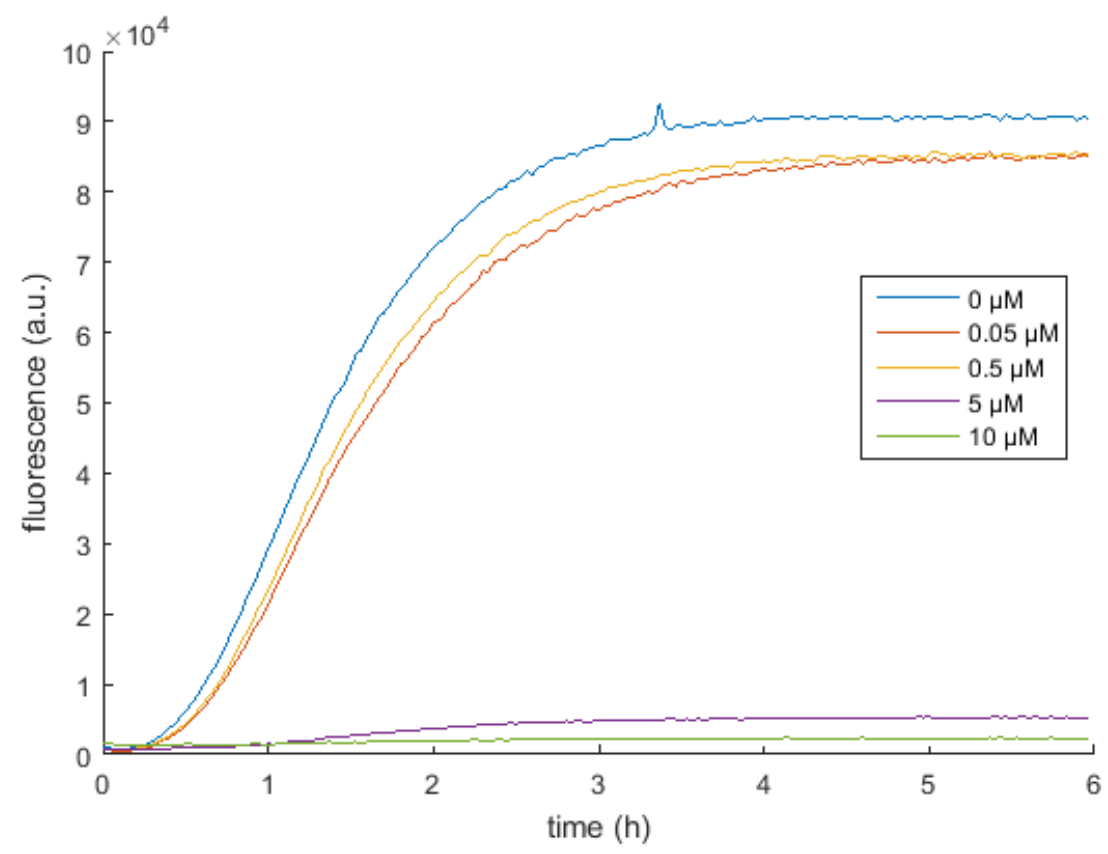

Figure S1. Cell-free expression of the fluorescence protein YPet in the presence of sDNA. Fluorescence measurement was carried out using a commercial plate reader. The concentrations used for the mRNAcomplementary sDNA were $0 \mu \mathrm{M}$ (cyan), $0.05 \mu \mathrm{M}$ (red), $0.5 \mu \mathrm{M}$ (yellow), $5 \mu \mathrm{M}$ (purple), $10 \mu \mathrm{M}$ (green). For the plasmid encoding YPet we used $5 \mathrm{nM}$ and the used promotor was $\mathrm{T7}$.

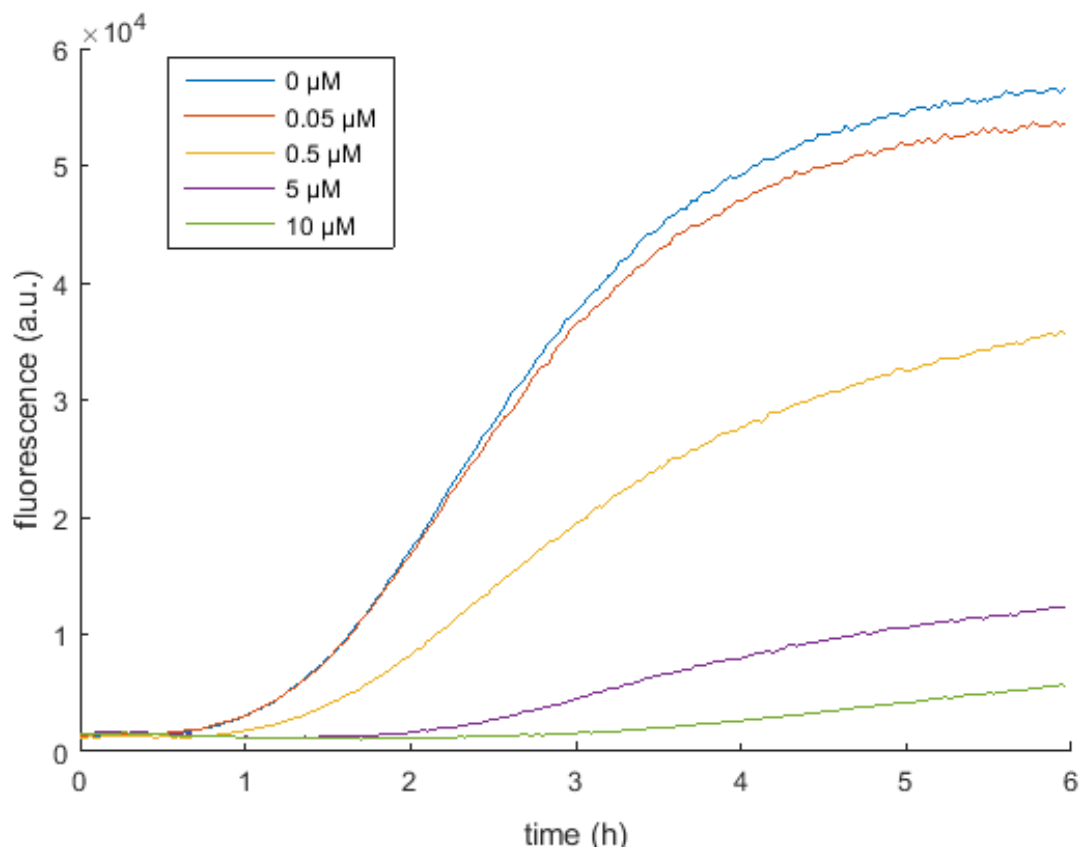

Figure S2. Cell-free expression of the fluorescence protein YPet in the presence of sDNA. Fluorescence measurement was carried out using a commercial plate reader. The concentrations used for the mRNAcomplementary sDNA were $0 \mu \mathrm{M}$ (cyan), $0.05 \mu \mathrm{M}$ (red), $0.5 \mu \mathrm{M}$ (yellow), $5 \mu \mathrm{M}$ (purple), $10 \mu \mathrm{M}$ (green). For the plasmid encoding YPet we used $5 \mathrm{nM}$ and the used promotor was a constitutive promotor (J23106). 


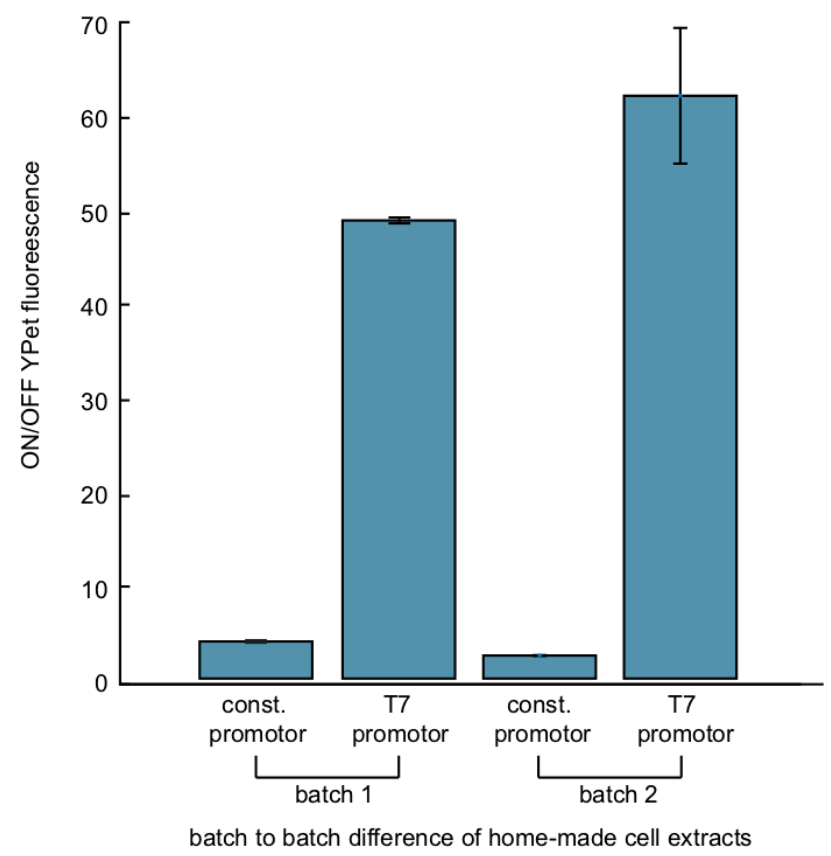

Figure S3. Comparison of ON/OFF YPet fluorescence for two different batches of home-made crude cell extracts. The lysis process was slightly different for both batches. The sDNA used had $60 \mathrm{nt}$ and a concentration of $10 \mu \mathrm{M}$. The given uncertainties are S.E.

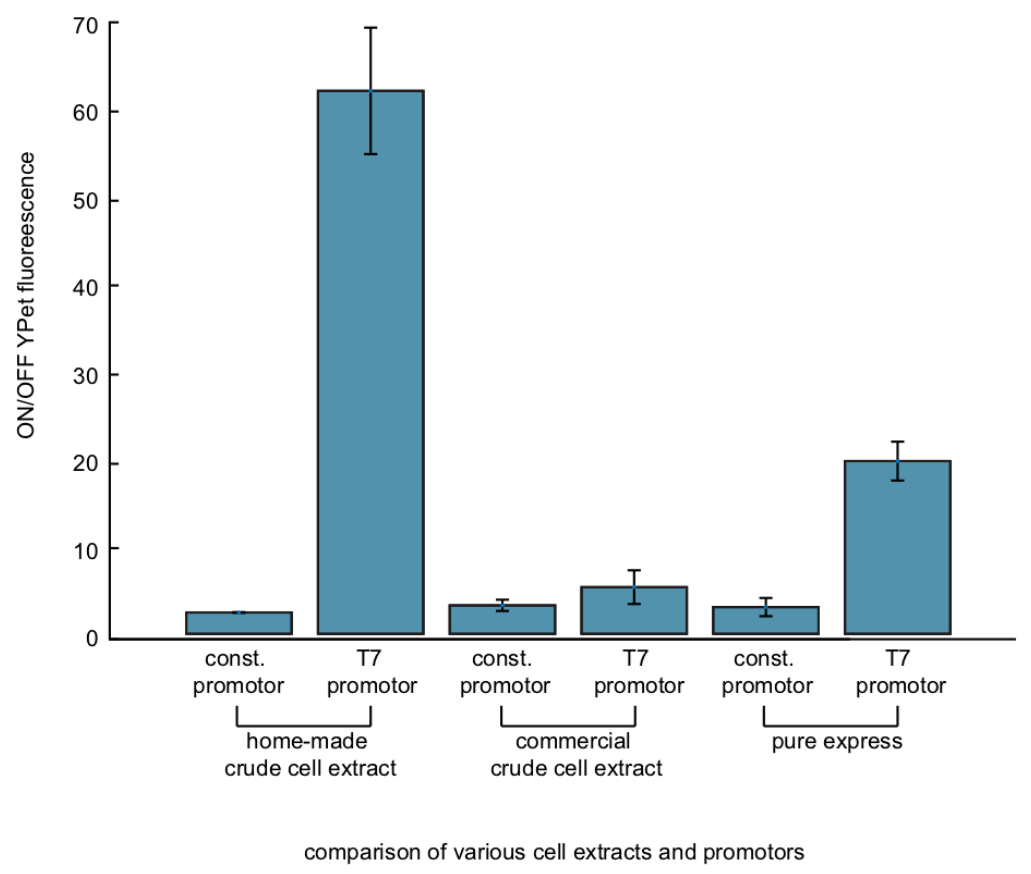

Figure S4. Comparison of ON/OFF YPet fluorescence for different cell free systems: a home-made crude cell extract, a commercially available crude cell extract (myTXTL from arbor biosciences) and the the PURExpress $\AA$ system from NEB. The sDNA used had $60 \mathrm{nt}$ and a concentration of $10 \mu \mathrm{M}$. The given uncertainties are S.E. 


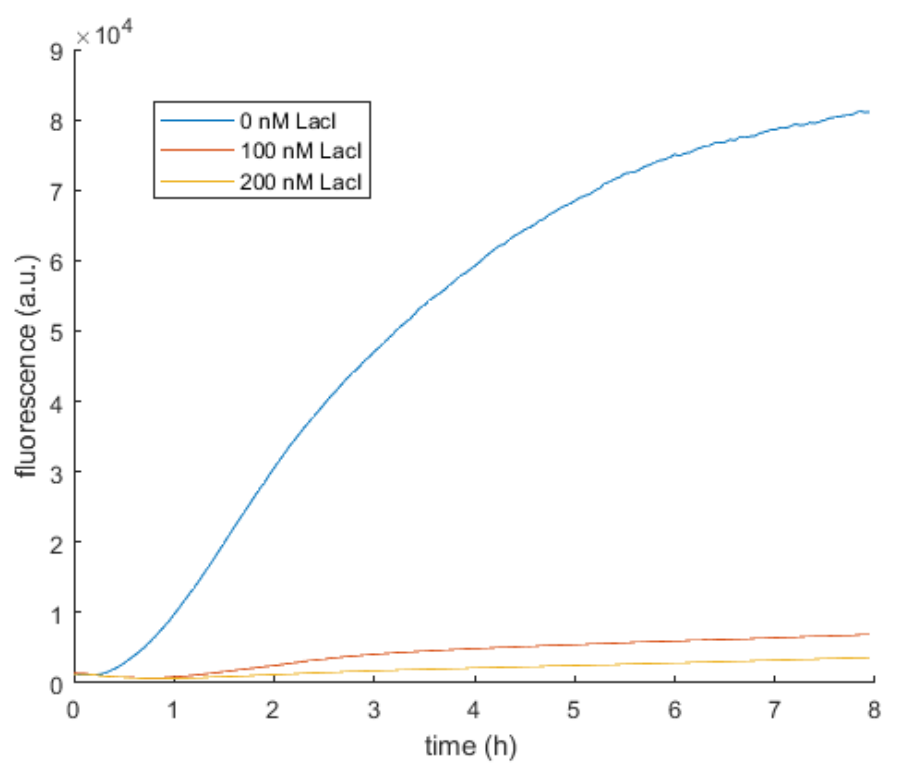

Figure S5. Cell-free expression of the fluorescence protein in the presence of Lacl. Fluorescence measurement was carried out using a commercial plate reader. The concentrations used for the Lacl were $0 \mathrm{nM}$ (cyan), $100 \mathrm{nM}$ (red), and $200 \mathrm{nM}$ (yellow). The fluorescence protein was controlled by a lactose operon at $5 \mathrm{nM}$ concentration. The ON/OFF ratio of the non-repressed signal to the repressed signal is for $100 \mathrm{nM} \mathrm{Lacl}$ approximatly 16.9 and for $200 \mathrm{nM}$ about 17.5 . 


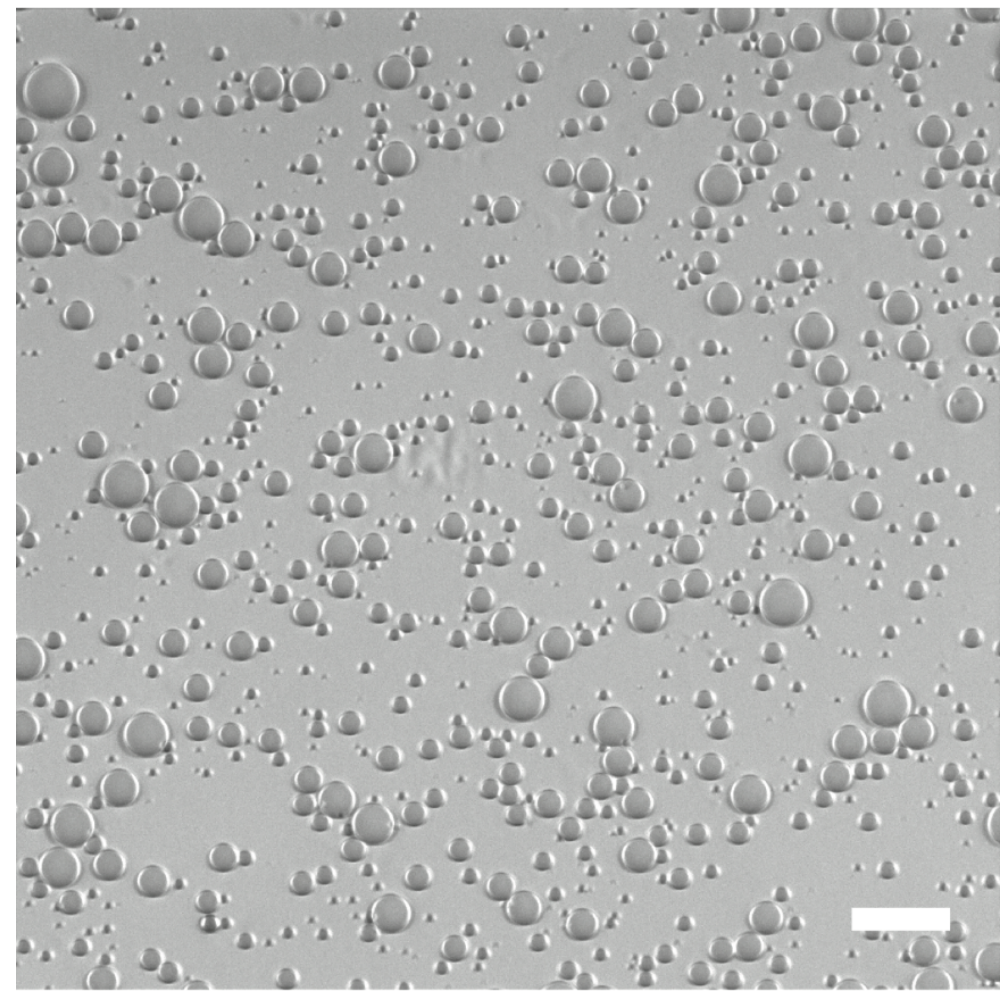

Figure S6. A typical micrograph of droplets created by shaking. Scale bar: $200 \mu \mathrm{m}$.

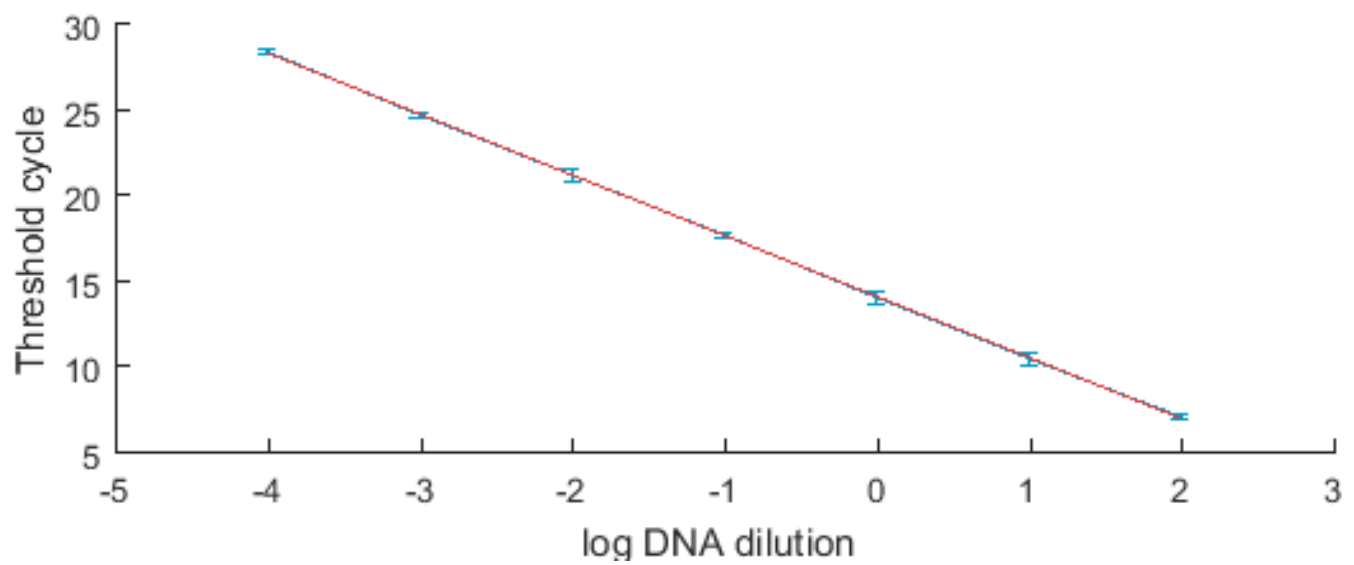

Figure S7. qPCR calibration curve to calculate the fold change of T7 DNA. The primer sequences used were ggacccacgtaaaccacatc and cattcggctaactccagagc. The linear fit of the threshold cycle vs. the log dilution of the T7 DNA resulted in an intersection with the $y$ axis at $14.1613 \mathrm{ct}$ and a slope of $-3.5507 \mathrm{ct}$. The $R^{2}$ coefficient was 0.9999 and the efficacy of the PCR reaction was $91.26 \%$. 


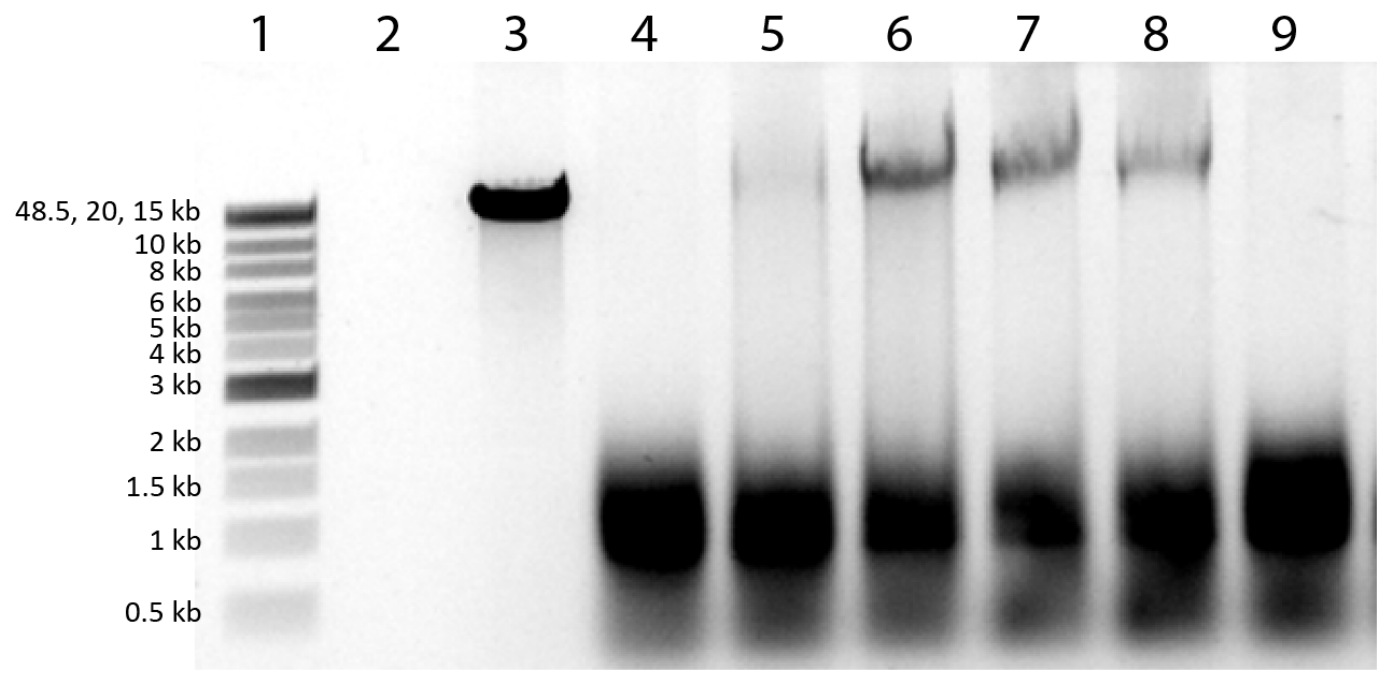

Figure S8. Agarose gel of the T7 DNA after several steps of dilution during the serial dilution experiments. From left to right: line 1, $1 \mathrm{~kb}$ extended ladder from NEB, lane 2, empty, lane 3, purified T7 DNA, lane 4, cell extract as a control, lane 5, cell extract and T7 DNA after $4^{\text {th }}$ incubation and dilution, lane 6, cell extract and T7 DNA after $3^{\text {rd }}$ incubation and dilution, lane 7, cell extract and T7 DNA after $2^{\text {nd }}$ incubation and dilution, lane 8, cell extract and T7 DNA after $1^{\text {st }}$ incubation and dilution, lane 9 , cell extract and T7 DNA before $1^{\text {st }}$ incubation.

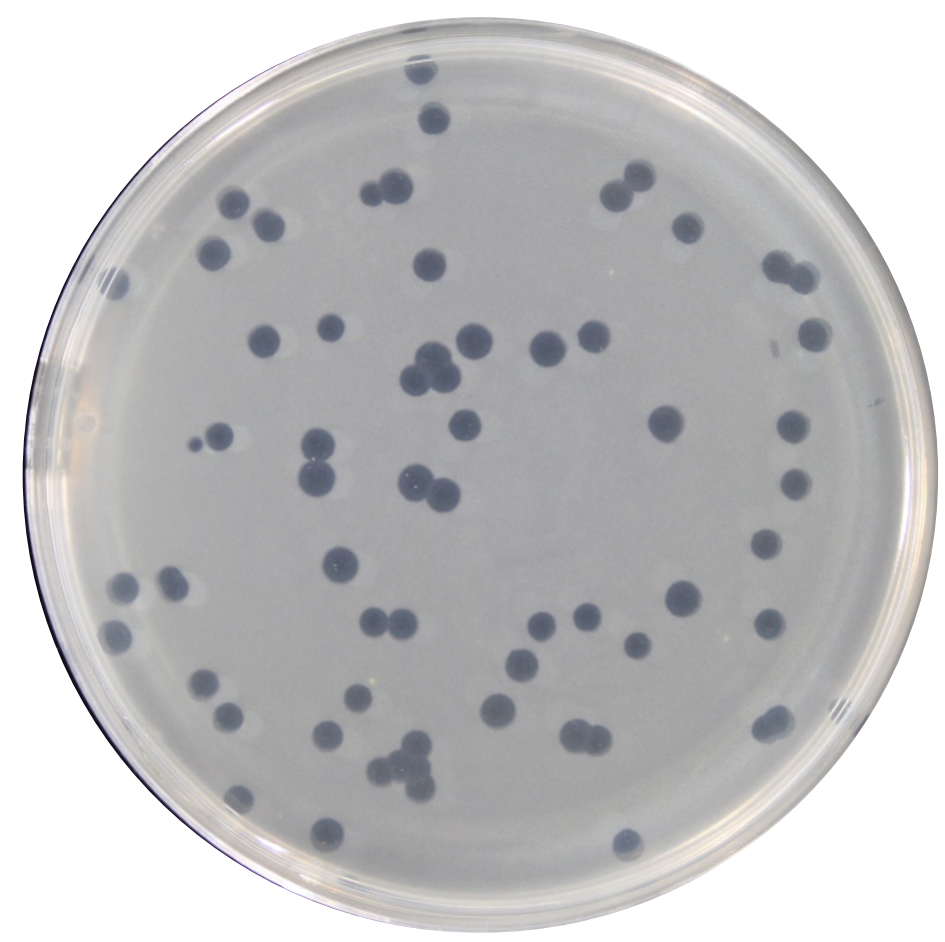

Figure S9. Example of a plaque assay of the cell-free produced $\mathrm{T} 7$ phage with $E$. coli to determine the phage titer of the sample. 


\section{DNA sequences}

\section{Plasmid sequences:}

pSB1C3 T7 YPet: gTAATACGACTCACTATAGGGTAGCGCAGCGCTCAACGGGTGTGCTTCCCGTTCTGATGAGTCCG TGAGGACGAAAGCGCCTCTACAAATAATTTTGTTTAATCATGAGaaagaggagaaaACTAGATGTCTA AAGGTGAAGAACTGTTTACGGGTGTCGTGCCGATTCTGGTCGAGTTGGACGGCGACGTGAACGG TCACAAATTCAGCGTGAGCGGCGAGGGCGAGGGTGACGCGACGTACGGTAAGCTGACTCTGAA GCTGCTGTGCACCACGGGTAAATTGCCGGTTCCGTGGCCGACCCTGGTCACGACGCTGGGTTAT GGTGTACAATGTTTTGCACGCTATCCGGACCACATGAAACAGCACGATTTCTTCAAGAGCGCGAT GCCGGAAGGCTATGTTCAGGAACGTACCATCTTTTTCAAAGATGATGGTAATTACAAAACCCGCG CAGAAGTGAAGTTCGAGGGTGACACCCTGGTGAACCGTATTGAGCTGAAGGGTATTGACTTCAA GGAAGATGGCAATATTCTGGGTCACAAACTGGAGTACAACTATAACAGCCATAACGTCTACATCA CCGCGGATAAGCAAAAAAATGGTATCAAAGCAAATTTCAAGATTCGCCACAACATCGAAGATGGC GGCGTGCAACTGGCCGATCATTATCAGCAGAATACCCCAATCGGTGACGGTCCGGTGCTGTTGC CGGATAACCACTACCTGAGCTATCAAAGCGCGTTGTTCAAAGACCCGAATGAAAAACGTGACCAC ATGGTTCTGCTGGAATTTCTGACCGCTGCGGGCATCACTGAAGGCATGAATGAACTGTACAAGAC GCGTGGTGGCGGCGGTTCGATGAGCAAGACTATCGTTTTGTCCGTCGGCGAGGCTACCCGTAC CTTGACCGAAATTCAATCCACCGCGGACCGTCAAATTTTTTGAGGAAAAAGTCGGTCCTCTGGTGG GTCGTCTGCGTCTGACCGCGAGCCTGCGCCAGAACGGTGCCAAAACGGCATACCGTGTTAATCT GAAACTGGATCAGGCCGACGTTGTGGACAGCGGTCTGCCGAAAGTCCGCTACACCCAGGTGTG GAGCCACGATGTGACGATCGTTGCGAATAGCACCGAAGCGAGCCGCAAGAGCCTGTACGACCT GACCAAGAGCCTGGTGGCAACGTCCCAAGTTGAAGATCTGGTTGTTAACCTGGTGCCGCTGGGT CGTTAAaGCATGCcggaGGAAACACAGAAAAAAGCCCGCACCTGACAGTGCGGGCTTTTTTTTTCG ACCAAAGGGGtgcAtactagtagcggccgctgcagtccggcaaaaaagggcaaggtgtcaccaccctgcccttttctttaaaaccgaa aagattacttcgcgttatgcaggcttcctcgctcactgactcgctgcgctcggtcgttcggctgcggcgagcggtatcagctcactcaaaggcggt aatacggttatccacagaatcaggggataacgcaggaaagaacatgtgagcaaaaggccagcaaaaggccaggaaccgtaaaaaggc cgcgttgctggcgttttccacaggctccgcccccctgacgagcatcacaaaaatcgacgctcaagtcagaggtggcgaaacccgacaggac tataaagataccaggcgtttccccctggaagctccctcgtgcgctctcctgttccgaccctgccgcttaccggatacctgtccgcctttctcccttcg ggaagcgtggcgcttctcatagctcacgctgtaggtatctcagttcggtgtaggtcgttcgctccaagctgggctgtgtgcacgaaccccccgttc agcccgaccgctgcgccttatccggtaactatcgtcttgagtccaacccggtaagacacgacttatcgccactggcagcagccactggtaaca ggattagcagagcgaggtatgtaggcggtgctacagagttcttgaagtggtggcctaactacggctacactagaagaacagtatttggtatctgc gctctgctgaagccagttaccttcggaaaaagagttggtagctcttgatccggcaaacaaaccaccgctggtagcggtggttttttgtttgcaagc agcagattacgcgcagaaaaaaaggatctcaagaagatcctttgatctttctacggggtctgacgctcagtggaacgaaaactcacgttaag ggattttggtcatgagattatcaaaaaggatcttcacctagatccttttaaattaaaaatgaagttttaaatcaatctaaagtatatatgagtaaacttg gtctgacagctcgaggcttggattctcaccaataaaaaacgcccggcggcaaccgagcgttctgaacaaatccagatggagttctgaggtcat tactggatctatcaacaggagtccaagcgagctcgatatcaaattacgccccgccctgccactcatcgcagtactgttgtaattcattaagcattct gccgacatggaagccatcacaaacggcatgatgaacctgaatcgccagcggcatcagcaccttgtcgccttgcgtataatatttgcccatggtg aaaacgggggcgaagaagttgtccatattggccacgtttaaatcaaaactggtgaaactcacccagggattggctgagacgaaaaacatatt ctcaataaaccctttagggaaataggccaggttttcaccgtaacacgccacatcttgcgaatatatgtgtagaaactgccggaaatcgtcgtggt attcactccagagcgatgaaaacgtttcagtttgctcatggaaaacggtgtaacaagggtgaacactatcccatatcaccagctcaccgtctttca ttgccatacgaaattccggatgagcattcatcaggcgggcaagaatgtgaataaaggccggataaaacttgtgcttattttctttacggtctttaaa aaggccgtaatatccagctgaacggtctggttataggtacattgagcaactgactgaaatgcctcaaaatgttctttacgatgccattgggatatat caacggtggtatatccagtgattttttctccatttagcttccttagctcctgaaaatctcgataactcaaaaaatacgcccggtagtgatcttatttcat tatggtgaaagttggaacctcttacgtgcccgatcaactcgagtgccacctgacgtctaagaaaccattattatcatgacattaacctataaaaat aggcgtatcacgagg cagaatttcagataaaaaaaatccttagcttcgctaaggatgatttctggaattcgAgTAAgccCCTCTAGAgg accacgcatcgtgatgcctatgcgcggtagtcccaccttgtccactagaatggaagattggcacgtatcaagactttggagtagtaccataacgc $\mathrm{c}$

pSB1C3 YPet: tttacggctagctcagtcctaggtatagtgctagcTAGCGCAGCGCTCAACGGGTGTGCTTCCCGTTCTGATGAGT CCGTGAGGACGAAAGCGCCTCTACAAATAATTTTGTTTAATCATGAGaaagaggagaaaACTAGATGT CTAAAGGTGAAGAACTGTTTACGGGTGTCGTGCCGATTCTGGTCGAGTTGGACGGCGACGTGAA CGGTCACAAATTCAGCGTGAGCGGCGAGGGCGAGGGTGACGCGACGTACGGTAAGCTGACTCT GAAGCTGCTGTGCACCACGGGTAAATTGCCGGTTCCGTGGCCGACCCTGGTCACGACGCTGGG TTATGGTGTACAATGTTTTGCACGCTATCCGGACCACATGAAACAGCACGATTTCTTCAAGAGCG CGATGCCGGAAGGCTATGTTCAGGAACGTACCATCTTTTTCAAAGATGATGGTAATTACAAAACC CGCGCAGAAGTGAAGTTCGAGGGTGACACCCTGGTGAACCGTATTGAGCTGAAGGGTATTGACT TCAAGGAAGATGGCAATATTCTGGGTCACAAACTGGAGTACAACTATAACAGCCATAACGTCTAC ATCACCGCGGATAAGCAAAAAAATGGTATCAAAGCAAATTTCAAGATTCGCCACAACATCGAAGA 
TGGCGGCGTGCAACTGGCCGATCATTATCAGCAGAATACCCCAATCGGTGACGGTCCGGTGCTG TTGCCGGATAACCACTACCTGAGCTATCAAAGCGCGTTGTTCAAAGACCCGAATGAAAAACGTGA CCACATGGTTCTGCTGGAATTTCTGACCGCTGCGGGCATCACTGAAGGCATGAATGAACTGTACA AGACGCGTGGTGGCGGCGGTTCGATGAGCAAGACTATCGTTTTTGTCCGTCGGCGAGGCTACCC GTACCTTGACCGAAATTCAATCCACCGCGGACCGTCAAATTTTTGAGGAAAAAGTCGGTCCTCTG GTGGGTCGTCTGCGTCTGACCGCGAGCCTGCGCCAGAACGGTGCCAAAACGGCATACCGTGTT AATCTGAAACTGGATCAGGCCGACGTTGTGGACAGCGGTCTGCCGAAAGTCCGCTACACCCAGG TGTGGAGCCACGATGTGACGATCGTTGCGAATAGCACCGAAGCGAGCCGCAAGAGCCTGTACG ACCTGACCAAGAGCCTGGTGGCAACGTCCCAAGTTGAAGATCTGGTTGTTAACCTGGTGCCGCT GGGTCGTTAAaGCATGCcggaGGAAACACAGAAAAAAGCCCGCACCTGACAGTGCGGGCTTTTTT TTTCGACCAAAGGGGtgcAtactagtagcggccgctgcagtccggcaaaaaagggcaaggtgtcaccaccctgcccttttctttaa aaccgaaaagattacttcgcgttatgcaggcttcctcgctcactgactcgctgcgctcggtcgttcggctgcggcgagcggtatcagctcactcaa aggcggtaatacggttatccacagaatcaggggataacgcaggaaagaacatgtgagcaaaaggccagcaaaaggccaggaaccgtaa aaaggccgcgttgctggcgtttttccacaggctccgcccccctgacgagcatcacaaaaatcgacgctcaagtcagaggtggcgaaacccga caggactataaagataccaggcgtttccccctggtttacggctagctcagtcctaggtatagtgctagcTAGCGCAGCGCTCAACGG GTGTGCTTCCCGTTCTGATGAGTCCGTaagctccctcgtgcgctctcctgttccgaccctgccgcttaccggatacctgtccgc ctttctcccttcgggaagcgtggcgctttctcatagctcacgctgtaggtatctcagttcggtgtaggtcgttcgctccaagctgggctgtgtgcacga accccccgttcagcccgaccgctgcgccttatccggtaactatcgtcttgagtccaacccggtaagacacgacttatcgccactggcagcagcc actggtaacaggattagcagagcgaggtatgtaggcggtgctacagagttcttgaagtggtggcctaactacggctacactagaagaacagta tttggtatctgcgctctgctgaagccagttaccttcggaaaaagagttggtagctcttgatccggcaaacaaaccaccgctggtagcggtggttttt tgtttgcaagcagcagattacgcgcagaaaaaaaggatctcaagaagatcctttgatctttctacggggtctgacgctcagtggaacgaaaac tcacgttaagggattttggtcatgagattatcaaaaaggatcttcacctagatccttttaaattaaaaatgaagttttaaatcaatctaaagtatatatg agtaaacttggtctgacagctcgaggcttggattctcaccaataaaaaacgcccggcggcaaccgagcgttctgaacaaatccagatggagtt ctgaggtcattactggatctatcaacaggagtccaagcgagctcgatatcaaattacgccccgccctgccactcatcgcagtactgttgtaattca ttaagcattctgccgacatggaagccatcacaaacggcatgatgaacctgaatcgccagcggcatcagcaccttgtcgccttgcgtataatattt gcccatggtgaaaacggggggcgaagaagttgtccatattggccacgtttaaatcaaaactggtgaaactcacccagggattggctgagacga aaaacatattctcaataaaccctttagggaaataggccaggtttcaccgtaacacgccacatcttgcgaatatatgtgtagaaactgccggaaa tcgtcgtggtattcactccagagcgatgaaaacgtttcagtttgctcatggaaaacggtgtaacaagggtgaacactatcccatatcaccagctc accgtctttcattgccatacgaaattccggatgagcattcatcaggcgggcaagaatgtgaataaaggccggataaaacttgtgcttattttcttta cggtctttaaaaaggccgtaatatccagctgaacggtctggttataggtacattgagcaactgactgaaatgcctcaaaatgttctttacgatgcca ttgggatatatcaacggtggtatatccagtgattttttctccattttagcttccttagctcctgaaaatctcgataactcaaaaaatacgcccggtagtg atcttatttcattatggtgaaagttggaacctcttacgtgcccgatcaactcgagtgccacctgacgtctaagaaaccattattatcatgacattaac ctataaaaataggcgtatcacgaggcagaatttcagataaaaaaaatccttagctttcgctaaggatgatttctggaattcgAgTAAgccCCT CTAGAggaccacgcatcgtgatgcctatgcgcggtagtcccaccttgtccactagaatggaagattggcacgtatcaagactttggagtagt accataacgccg

\section{sDNA sequences:}

40nt YPet: AGACATCTAGTttctcctcttCTCATGATTAAACAAAA

50nt YPet: CTTCACCTTTAGACATCTAGTtttctcctctttCTCATGATTAAACAAAA

60nt YPet:

GTAAACAGTTCTTCACCTTTAGACATCTAGTtttctcctcttCTCATGATTAAACAAAA

T7 phi10:

AGCCATATGTATATCTCCTTCTTAAAGTTAAACAAAATTATTTCTAGAGGGAAACCGTTG

qPCR Primer:

ggacccacgtaaaccacatc

cattcggctaactccagagc 


\section{DNA and RNA structure analysis}

We used the NUPACK Web Application to determine the secondary structure of the sDNA used. For the RNA structure analysis and for the calculation of the minimum free energy (MFE) of RNA-DNA conjugation we used the forna Server and the RNAcofold Server from the ViennaRNA Web Services (rna.tbi.univie.ac.at). In order to compare the stability of different sequences we altered the mRNA sequences for the calculations by poly-G resp. poly-U (Table S1). Table S2 shows the frequency of the MFE structures, DG and the single free energies of the monomers, homodimers and heterodimer of various ensembles.

\begin{tabular}{|c|c|c|}
\hline & Description/name & sequence \\
\hline Ynet & mRNA Ynet short & $\begin{array}{l}\text { GAAAGCGCCUCUACAAAUAAUUUUGUUUAAU } \\
\text { CAUGAGaaagaggagaaaACUAGAUGUCUAAAG } \\
\text { GUGAAGAACUGUUUACGGGUGUCGUGCCG }\end{array}$ \\
\hline YPet G & mRNA YPet short Poly-G & $\begin{array}{l}\text { GAAAGCGCCUCUACAAAUAAUUUUGGGGGG } \\
\text { GGGGGGGaaagaggagaaaACUAGAUGUCUAAA } \\
\text { GGUGAAGAACUGUUUACGGGUGUCGUGCCG }\end{array}$ \\
\hline YPet U & mRNA YPet short Poly-U & $\begin{array}{l}\text { GAAAGCGCCUCUACAAAUAAUUUUUUUUUUU } \\
\text { UUUUUUaaagaggagaaaACUAGAUGUCUAAAG } \\
\text { GUGAAGAACUGUUUACGGGUGUCGUGCCG }\end{array}$ \\
\hline 40 Ypet & sDNA 40 nt Ypet & $\begin{array}{l}\text { AGACATCTAGTtttctcctctttCTCATGATTAAACAA } \\
\text { AA }\end{array}$ \\
\hline 40 Ypet $C$ & sDNA 40 nt Ypet Poly-C & $\begin{array}{l}\text { AGACATCTAGTtttctcctctttCCCCCCCCCCCCCA } \\
\text { AAA }\end{array}$ \\
\hline 40 Ypet $\mathrm{A}$ & sDNA 40 nt Ypet Poly-A & $\begin{array}{l}\text { AGACATCTAGTtttctcctctttAAAAAAAAAAAAAAA } \\
\text { AA }\end{array}$ \\
\hline 50 Ypet & sDNA 50 nt Ypet & $\begin{array}{l}\text { CTTCACCTTTAGACATCTAGTtttctcctctttCTCAT } \\
\text { GATTAAACAAAA }\end{array}$ \\
\hline 50 Ypet $\mathrm{C}$ & sDNA 50 nt Ypet Poly-C & $\begin{array}{l}\text { CTTCACCTTTAGACATCTAGTtttctcctctttCCCCC } \\
\text { CCCCCCCCAAAA }\end{array}$ \\
\hline 50 Ypet A & sDNA 50 nt Ypet Poly-A & $\begin{array}{l}\text { CTTCACCTTTAGACATCTAGTtttctcctctttAAAAA } \\
\text { AAAAAAAAAAAA }\end{array}$ \\
\hline 60 Ypet & sDNA 60 nt Ypet & $\begin{array}{l}\text { GTAAACAGTTCTTCACCTTTAGACATCTAGTtttc } \\
\text { tcctctttCTCATGATTAAACAAAA }\end{array}$ \\
\hline 60 Ypet $\mathrm{C}$ & sDNA 60 nt Ypet Poly-C & $\begin{array}{l}\text { GTAAACAGTTCTTCACCTTTAGACATCTAGTtttc } \\
\text { tcctctttCCCCCCCCCCCCCAAAA }\end{array}$ \\
\hline 60 Ypet A & sDNA 60 nt Ypet Poly-A & $\begin{array}{l}\text { GTAAACAGTTCTTCACCTTTAGACATCTAGTtttc } \\
\text { tcctctttAAAAAAAAAAAAAAAAA }\end{array}$ \\
\hline $\mathrm{mcp}$ & $\begin{array}{l}\text { mRNA major capsid protein } \\
\text { short }\end{array}$ & $\begin{array}{l}\text { gggagaccacaacggUUUcccUcUagaaaUaaUUUUg } \\
\text { UUUaacUUUaagaaggagaUaUacaUaUggcUagca } \\
\text { UgacUggUggacagcaaaUg }\end{array}$ \\
\hline T7_phi10 & sDNA T7_phi10 & $\begin{array}{l}\text { AGCCATATGTATATCTCCTTCTTAAAGTTAAAC } \\
\text { AAAATTATTTCTAGAGGGAAACCGTTG }\end{array}$ \\
\hline
\end{tabular}

Table S1. Overview of DNA and RNA sequences used for calculations. 


\begin{tabular}{|c|c|c|c|c|c|c|c|c|}
\hline$A$ & B & $\begin{array}{l}\text { Frequ. } \\
\text { MFE } \\
\text { Struct. }\end{array}$ & $\begin{array}{c}\Delta G \\
\text { hetero- } \\
\text { dimer }\end{array}$ & $\begin{array}{c}\mathrm{AB} \\
\text { hetero- } \\
\text { dimer }\end{array}$ & $\begin{array}{c}\text { AA } \\
\text { homo- } \\
\text { dimer }\end{array}$ & $\begin{array}{c}\text { BB } \\
\text { homo- } \\
\text { dimer }\end{array}$ & $\begin{array}{c}\text { A } \\
\text { mono- } \\
\text { mer }\end{array}$ & $\begin{array}{c}\text { B } \\
\text { mono- } \\
\text { mer }\end{array}$ \\
\hline Ypet & 40 Ypet & $8.25 \%$ & -54.78 & -70.14 & -37.14 & -7.92 & -14.04 & -1.32 \\
\hline Ypet & 50 Ypet & $25.12 \%$ & -70.94 & -86.75 & -37.14 & -9.12 & -14.04 & -1.77 \\
\hline Ypet & 60 Ypet & $24.98 \%$ & -86.73 & -103.65 & -37.14 & -11.22 & -14.04 & -2.88 \\
\hline YPet G & 40 Ypet C & $8.34 \%$ & -72.74 & -90.23 & -42.16 & -5.55 & -17.00 & -0.49 \\
\hline YPet G & 50 Ypet C & $25.40 \%$ & -88.88 & -106.84 & -42.16 & -7.12 & -17.00 & -0.96 \\
\hline YPet G & 60 Ypet C & $25.26 \%$ & -104.42 & -123.75 & -42.16 & -7.45 & -17.00 & -2.33 \\
\hline YPet U & 40 Ypet A & $7.86 \%$ & -40.38 & -59.17 & -43.53 & -5.68 & -18.22 & -0.57 \\
\hline YPet U & 50 Ypet A & $23.94 \%$ & -56.44 & -75.78 & -43.53 & -7.32 & -18.22 & -1.13 \\
\hline YPet U & 60 Ypet A & $23.80 \%$ & -72.06 & -92.68 & -43.53 & -7.78 & -18.22 & -2.04 \\
\hline YPet G & 40 Ypet C & $8.34 \%$ & -72.74 & -90.23 & -42.16 & -5.55 & -17.00 & -0.49 \\
\hline Ypet & 40 Ypet & $8.25 \%$ & -54.78 & -70.14 & -37.14 & -7.92 & -14.04 & -1.32 \\
\hline YPet U & 40 Ypet A & $7.86 \%$ & -40.38 & -59.17 & -43.53 & -5.68 & -18.22 & -0.57 \\
\hline YPet G & 50 Ypet C & $25.40 \%$ & -88.88 & -106.84 & -42.16 & -7.12 & -17.00 & -0.96 \\
\hline Ypet & 50 Ypet & $25.12 \%$ & -70.94 & -86.75 & -37.14 & -9.12 & -14.04 & -1.77 \\
\hline YPet U & 50 Ypet A & $23.94 \%$ & -56.44 & -75.78 & -43.53 & -7.32 & -18.22 & -1.13 \\
\hline YPet G & 60 Ypet C & $25.26 \%$ & -104.42 & -123.75 & -42.16 & -7.45 & -17.00 & -2.33 \\
\hline Ypet & 60 Ypet & $24.98 \%$ & -86.73 & -103.65 & -37.14 & -11.22 & -14.04 & -2.88 \\
\hline YPet U & 60 Ypet A & $23.80 \%$ & -72.06 & -92.68 & -43.53 & -7.78 & -18.22 & -2.04 \\
\hline Ypet & 60 Ypet & $24.98 \%$ & -86.73 & -103.65 & -37.14 & -11.22 & -14.04 & -2.88 \\
\hline Ypet & 60 Ypet C & $26.54 \%$ & -65.85 & -82.22 & -37.14 & -7.45 & -14.04 & -2.33 \\
\hline Ypet & 60 Ypet A & $10.95 \%$ & -63.52 & -79.96 & -37.14 & -7.78 & -14.04 & -2.4 \\
\hline Ypet & 40 Ypet & $8.25 \%$ & -54.78 & -70.14 & -37.14 & -7.92 & -14.04 & -1.32 \\
\hline Ypet & 40 Ypet C & $8.76 \%$ & -34.17 & -48.7 & -37.14 & -5.55 & -14.04 & -0.49 \\
\hline Ypet & 40 Ypet A & $3.62 \%$ & -31.84 & -46.45 & -37.14 & -5.68 & -14.04 & -0.57 \\
\hline Ypet & 60 Ypet & $24.98 \%$ & -86.73 & -103.65 & -37.14 & -11.22 & -14.04 & -2.88 \\
\hline $\mathrm{mcp}$ & T7_phi10 & $39.55 \%$ & -71.8 & -105.07 & -52.54 & -24.69 & -23.63 & -9.64 \\
\hline
\end{tabular}

Table S2. Overview of Results from calculations using ViennaRNA Web Services RNAcofold server. 


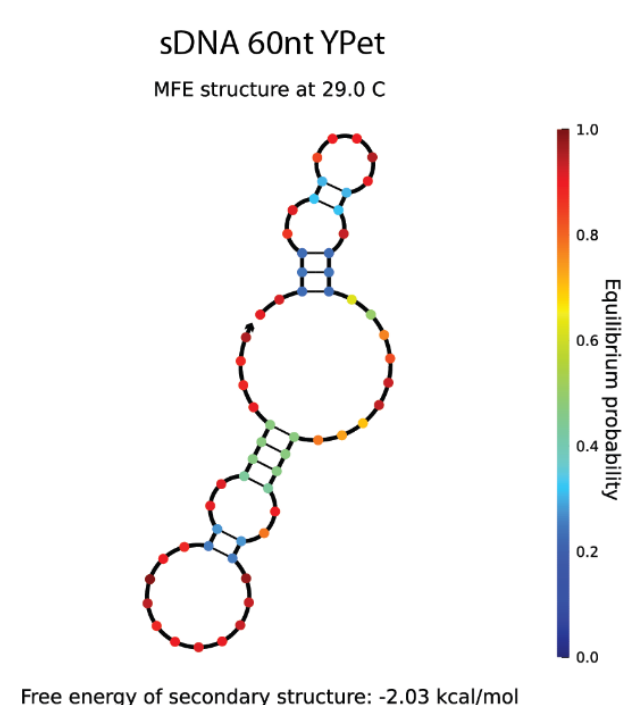

Free energy of secondary structure: $-2.03 \mathrm{kcal} / \mathrm{mol}$

\section{sDNA 40nt YPet}

MFE structure at $29.0 \mathrm{C}$

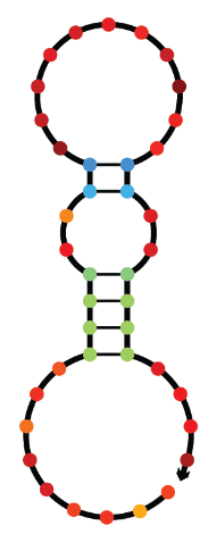

Free energy of secondary structure: $-1.58 \mathrm{kcal} / \mathrm{mol}$

\section{sDNA T7_phi10}

MFE structure at $29.0 \mathrm{C}$

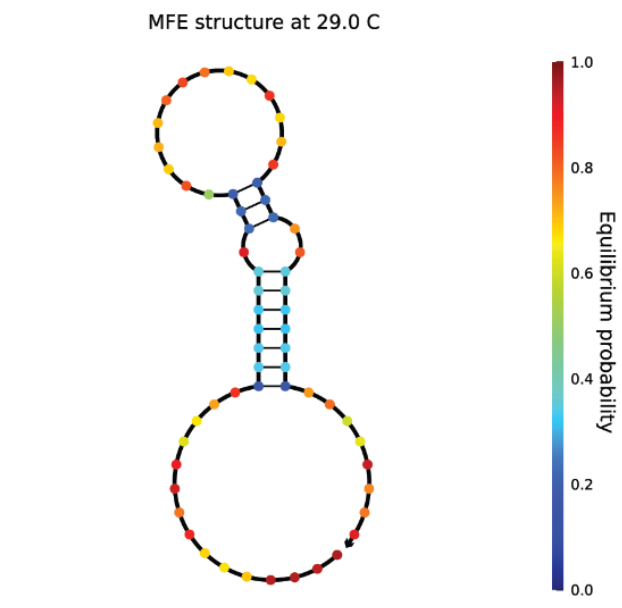

Free energy of secondary structure: $-2.81 \mathrm{kcal} / \mathrm{mol}$
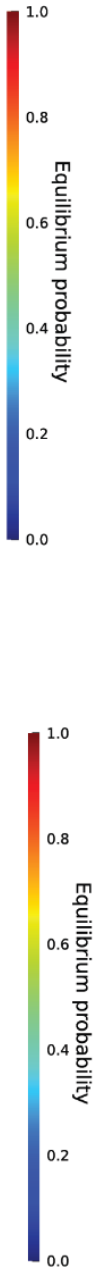

SDNA 50nt YPet

MFE structure at $29.0 \mathrm{C}$

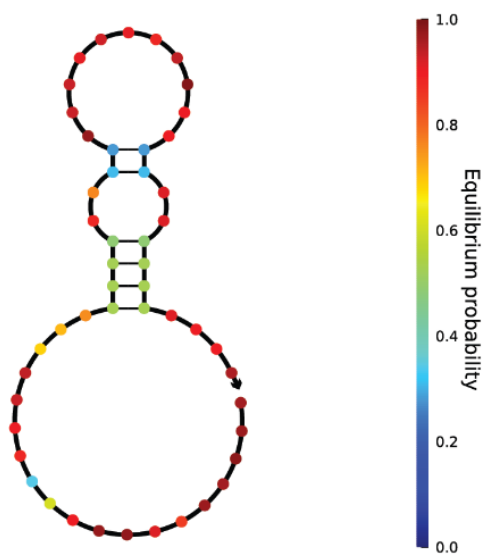

Free energy of secondary structure: $-1.58 \mathrm{kcal} / \mathrm{mol}$

mRNA YPet short

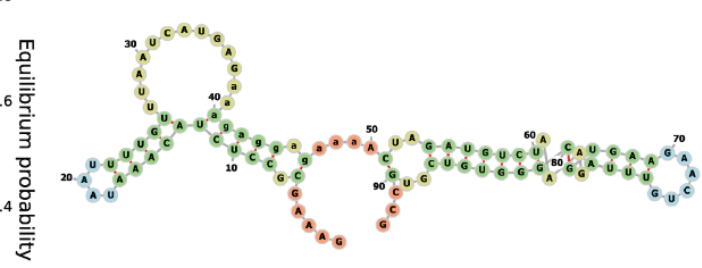

mRNA major capsid protein short

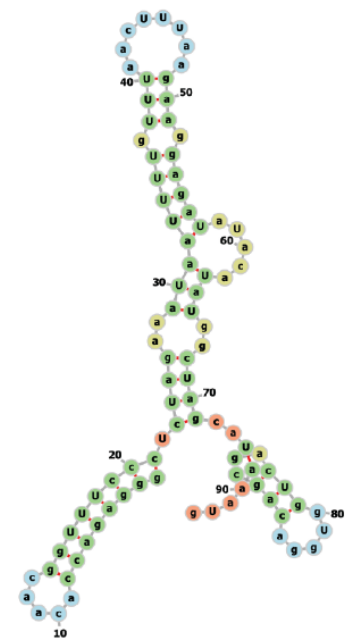

Figure S10. Overview of sDNA (NUPACK) and mRNA (ViennaRNA Web Services forna Server) secondary structures. 